
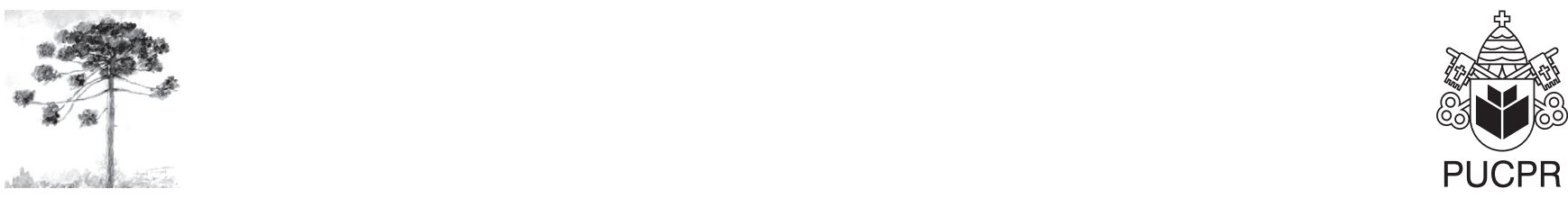

PUCPR

\title{
Registro de cardume misto de espécies da família Haemulidae nas Ilhas Itacolomis, Santa Catarina, Brasil
}

\author{
Record of mixed-species shoal of Haemulidae family in \\ Itacolomis Islands, Santa Catarina, Brazil
}

\author{
Marcelo Rennó Braga ${ }^{[a]}$, Raul Rennó Braga ${ }^{[b]}$, José Marcelo Rocha Aranha ${ }^{[c]}$
}

[a] Professor Doutor do Instituto Federal de Educação, Ciência e Tecnologia de Santa Catarina (IF-SC), Florianópolis - Brasil, e-mail: marcelo.braga@ifsc.edu.br

[b] Bacharel em Biologia da Pontifícia Universidade Católica do Paraná (PUC-PR), Curitiba, PR - Brasil, e-mail: raulbraga@onda.com.br

[c] Professor Doutor do Laboratório de Ecologia de Rios, Universidade Federal do Paraná (UFPR), Curitiba, PR - Brasil, e-mail: jmaranha@ufpr.br

\section{Resumo}

Este trabalho registra a ocorrência de cardume misto formado por três espécies de peixes da família Haemulidae: Haemulon aurolineatum (Cuvier, 1830), Haemulon steindachneri (Jordan \& Gilbert, 1882) e Orthopristis ruber (Cuvier, 1830). O comportamento foi constatado nas Ilhas Itacolomis, localizadas no litoral norte de Santa Catarina, por meio de observação direta em mergulho autônomo. Dentre os benefícios envolvidos na formação de cardumes, destacam-se a maior proteção contra predadores e a maior habilidade no forrageamento. Contrapondo tais benefícios, em cardumes mistos pode ocorrer aumento da competição quando as espécies envolvidas possuem nichos alimentares semelhantes, como as espécies envolvidas neste registro. Nesse caso, os benefícios provenientes da formação do cardume misto devem estar relacionados à abundância de cada espécie e à presença de predadores.

Palavras-chave: Cardume misto. Peixes recifais. Comportamento.

\section{Abstract}

This scientific note registers the occurrence of mixed-species shoal composed by three species of fish that belong to Haemulidae family: Haemulon aurolineatum (Cuvier, 1830) Haemulon steindachneri (Jordan \& Gilbert, 1882) 
and Orthopristis ruber (Cuvier, 1830). The behavior was registered by direct observation during a SCUBA dive at Itacolomis Islands, which are located in the north coast of Santa Catarina State. Among the benefits involving a shoal formation is the higher protection from predators and higher foraging ability. Regardless form those benefits, mixed-shoals can increase competition when involved species have similar dietary niche like the ones registered. On this special case the benefits involved might be related to species abundance and to predators.

Keywords: Mixed-species shoal. Reef-fish. Behavior.

A formação de cardumes tem sido foco de diversos estudos ecológicos, comportamentais e evolucionários. De acordo com Pitcher e Parrish (1), nas décadas de 1960 e 1970 houve uma tendência em estudar aspectos como homogeneidade, sincronia e estrutura tridimensional de cardumes. A partir da década de 1980, grande parte dos estudos investigou os custos e benefícios de um indivíduo pertencer a um grupo ou cardume. No Brasil, o assunto tem sido pouco estudado e a maior parte das abordagens ocorre no formato de notas científicas ou breve comunicações. Dessa forma, destacam-se: a investigação sobre a formação de cardume misto de Mulloidichthys martinicus (Cuvier, 1829) e Haemulon chrysargyreum (Günther, 1859), com função de mimetismo de proteção, realizada por Krajewski et al. (2). Queiroz e Magurran (3) enfatizaram a proteção contra predadores em cardumes de Pygocentrus nattereri (Kner, 1858) e Carvalho et al. (4) estudaram o comportamento de formação de cardume de Mugil curema (Valenciennes, 1836), em Ubatuba, São Paulo.

De forma geral, a proteção contra predadores e o aumento na habilidade de forrageamento são descritos como os fatores determinantes nas decisões de um determinado indivíduo participar ou não de um cardume $(1,5,6)$. No caso de cardumes mistos, a maioria dos fatores que favorecem a formação dos cardumes de uma única espécie também se aplica à formação dos cardumes formados por indivíduos de espécies diferentes (7). Os benefícios do forrageamento mais efetivo e da melhor vigilância contra predadores são provavelmente maiores quanto maior for a similaridade morfológica e da dieta dos diferentes participantes. Contrapondo tais benefícios, a formação de cardumes provoca o aumento da competição entre indivíduos e, de acordo com Pitcher e Parrish (1), tal custo pode ser diminuído no caso dos cardumes mistos.

As observações deste estudo foram realizadas nas Ilhas Itacolomis, localizadas no município de Piçarras, litoral norte de Santa Catarina, e suas coordenadas são: 26²42'38.2"S/48³7'04.8"W. As três espécies observadas: Haemulon aurolineatum (Cuvier, 1830), Haemulon steindachneri (Jordan \& Gilbert, 1882) e Orthopristis ruber (Cuvier, 1830) pertencem à família Haemulidae. A espécie H. aurolineatumatinge em torno de $25 \mathrm{~cm}$ de comprimento, habita fundos arenosos, bancos de gramíneas marinhas e ambientes recifais de até 40 metros de profundidade; forma grandes cardumes e se alimenta de pequenos crustáceos, moluscos, outros invertebrados, plâncton e algas; ocorre no Oceano Atlântico - de Massachusetts (EUA) até o Sul do Brasil $(8,9)$. A espécie $H$. steindachneri atinge em torno de $30 \mathrm{~cm}$, habita ambientes recifais e arenosos até 30 metros de profundidade, alimenta-se de invertebrados bentônicos e ocorre no Oceano Atlântico - do Panamá até o Sul do Brasil-, sendo também registrada no Oceano Pacífico - do México ao Peru $(8,9)$. A espécie $O$. ruber atinge até $40 \mathrm{~cm}$ de comprimento e habita fundos rochosos e de areia até 70 metros de profundidade. Tal espécie alimenta-se de crustáceos e outros invertebrados e ocorre no Oceano Atlântico - de Honduras até o Sul do Brasil $(8,9)$. Diferentes autores descrevem as espécies mencionadas como formadoras de cardumes mistos que usualmente são compostos por espécies da mesma família (Haemulidae) ou da família Lutjanidae $(9,10)$, no entanto, não são mencionadas quais espécies participam dessas associações.

A observação do cardume misto ocorreu durante uma das fases de campo de um estudo com amostragens mensais entre janeiro de 2005 e janeiro de 2007. Durante esse período, as três espécies de Haemulidae mencionadas foram sempre abundantes, no entanto, a observação de cardume misto ocorreu apenas uma vez, no dia 21/02/2006, em mergulho realizado na face leste das Ilhas Itacolomis. O registro ocorreu aos 10 metros de profundidade sobre fundo mesclado de areia e rochas. O cardume era composto por 15 indivíduos com comprimentos 
totais entre 13 e $25 \mathrm{~cm}$, sendo um $H$. steindachneri, quatro $H$. aurolineatum e dez O. rubra.

De acordo com Ward et al. (11), um cardume misto pode diminuir o custo da competição entre indivíduos quando os recursos explorados são diferentes. O nicho alimentar das espécies analisadas é semelhante, portanto, a redução da competição não deve ser um dos fatores preponderantes na formação do cardume misto observado. Variações de condições bióticas como abundância de cada espécie de Haemulidae e presença de predadores, possivelmente elevam os benefícios da formação do cardume misto. As condições ideais para formação desses agrupamentos podem ser efêmeras, o que explica uma única observação de cardume misto em dois anos de observações mensais. Por fim, este registro exemplifica e enfatiza a plasticidade do comportamento de formação de cardumes.

\section{Referências}

1. PicherTJ, ParrishJK. Function of shoaling behaviour in teleosts. In: Picher TJ. Behaviour of teleost fishes. 2nd ed. London: Chapman \& Hall; 1993. p. 363-439.

2. Krajewski JP, Bonaldo RM, Sazima C, Sazima I. The association of the goatfish Mulloidichthys martinicus with the grunt Haemulon chrysargyreum: an example of protective mimicry. Biota Neotropica 2004;4(1):1-4.

3. Queiroz H, Magurran AE. Safety in numbers? Shoaling behaviour of the Amazonian red-bellied piranha. Biol Lett. 2005;1(2):155-7.

4. Carvalho CD, Corneta CM, Uieda VS. Schooaling behavior of Mugil curema (Perciformes: Mugilidae) in an estuary in southeastern Brazil. Neotropical Ichthyology. 2005;5(1):81-3.
5. TienJH, Simon AL, Rubenstein DI. Dynamics of fish shoals: identifying key decision rules. Evolutionary Ecology Research. 2004;6:555-65.

6. Krebs JR, Davies NB. Introdução à ecologia comportamental. São Paulo: Atheneu; 1996.

7. Ehrlich PR, Ehrlich AH. Coevolution: heterotypic schooling in Caribbean reef fishes. Am Naturalist. 1973;107:157-60.

8. Carpenter KE. The living marine resources of the Western Central Atlantic. Volume 3: bony fishes part 2 (Opistognathidae to Molidae), sea turtles and marine mammals. FAO Species Identification Guide for Fishery Purposes and American Society of Ichthyologists and Herpetologists Special Publication N. 5. Rome: FAO; 2002. p. 1375-2127.

9. Hostim-Silva M, Andrade AB, Machado LF, Gerhardinger LC, Daros FA, Barreios JP, et al. Peixes de Costão Rochoso de Santa Catarina: I. Arvoredo. Itajaí: Universidade do Vale do Itajaí; 2006.

10. Humann P, Deloach N. Reef fish identification: Florida, Caribbean, Bahamas. 3rd ed. Jacksonville: New World Publications; 2002.

11. Ward AJW, Axford S, Krause J. Mixed-species shoaling in fish: the sensory mechanisms and costs of shoal choice. Behav Ecol Sociobiol. 2002;52:182-7.

Recebido: 17/02/2009

Received: 02/17/2009

Aprovado: 14/10/2009

Approved: 10/14/2009 